SUBJECT AREAS:

PROSTATE CANCER

CANCER GENETICS

DIAGNOSTIC MARKERS

BIOMARKER RESEARCH

Received

10 October 2012

Accepted

3 June 2013

Published

24 June 2013

Correspondence and requests for materials should be addressed to E.K. (emma.killick@icr. ac.uk)

* Joint last authors.

\section{Role of Engrailed-2 (EN2) as a prostate cancer detection biomarker in genetically high risk men}

Emma Killick', Richard Morgan ${ }^{3}$, Francesca Launchbury ${ }^{3}$, Elizabeth Bancroft ${ }^{2}$, Elizabeth Page', Elena Castro' ', Zsofia Kote-Jarai ${ }^{1}$, Armen Aprikian ${ }^{4}$, Ignacio Blanco ${ }^{5}$, Virginia Clowes ${ }^{6}$, Susan Domchek7, Fiona Douglas ${ }^{8}$, Diana Eccles 9 , D. Gareth Evans ${ }^{10}$, Marion Harris ${ }^{11}$, Judy Kirk ${ }^{12}$, Jimmy Lam ${ }^{13}$, Geoffrey Lindeman ${ }^{14}$, Gillian Mitchell ${ }^{15}$, Nicholas Pachter ${ }^{16}$, Christina Selkirk ${ }^{17}$, Kathy Tucker $^{18}$, Janaz Zgajnar ${ }^{19}$, Rosalind Eeles ${ }^{1,2 *}$ \& Hardev Pandha ${ }^{3 *}$

\footnotetext{
${ }^{1}$ Institute of Cancer Research, Sutton, Surrey, UK, ${ }^{2}$ Royal Marsden NHS Foundation Trust, London, UK, ${ }^{3}$ University of Surrey, Guildford, UK, ${ }^{4} \mathrm{McGill}$ University, Montreal, Canada, ${ }^{5}$ Catalonian Institute of Oncology, Barcelona, Spain, ${ }^{6}$ Addenbrookes Hospital, Cambridge, UK, ${ }^{7}$ University of Pennsylvania, Philadelphia, PA, USA, ${ }^{8}$ Institute of Human Genetics, Newcastle, UK, ${ }^{9}$ Wessex Clinical Genetics Service, Southampton, UK, ${ }^{10}$ Central Manchester University Hospitals NHS Foundation Trust, Manchester, UK, ${ }^{11}$ Monash Medical Centre, Melbourne, Australia, ${ }^{12}$ Westmead Hospital, Sydney, Australia, ${ }^{13}$ Repatriation General Hospital, Adelaide, Australia, ${ }^{14}$ The Royal Melbourne Hospital and The Walter \& Eliza Hall Institute of Medical Research, Melbourne, Australia, ${ }^{15}$ Peter MacCallum Cancer Centre, Melbourne, Australia and the Sir Peter MacCallum Department of Oncology, The University of Melbourne, Melbourne, Australia, ${ }^{16}$ King Edward Memorial Hospital, Perth, Australia, ${ }^{17}$ NorthShore University HealthSystem, Evanston, IL, USA, ${ }^{18}$ Prince of Wales Hospital, Sydney, Australia, ${ }^{19}$ Institute of Oncology, Liubljana, Slovenia.
}

Controversy surrounds the use of PSA as a biomarker for prostate cancer detection, leaving an unmet need for a novel biomarker in this setting; urinary EN2 may identify individuals with clinically relevant prostate cancer. Male $B R C A 1$ and $B R C A 2$ mutation carriers are at increased risk of clinically significant prostate cancer and may benefit from screening. Urine samples from $413 B R C A 1$ and BRCA2 mutation carriers and controls were evaluated. Subjects underwent annual PSA screening with diagnostic biopsy triggered by PSA $>3.0 \mathrm{ng} / \mathrm{ml} ; 21$ men were diagnosed with prostate cancer. Urinary EN2 levels were measured by ELISA and had a sensitivity of $66.7 \%$ and specificity of $89.3 \%$ for cancer detection. There was no statistically significant difference in EN2 levels according to genetic status or Gleason score. Urinary EN2 may be useful as a non-invasive early biomarker for prostate cancer detection in genetically high-risk individuals.

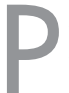

rostate cancer (PC) is the most commonly diagnosed male cancer in the US and the second most common cause of cancer death among males ${ }^{1}$. Men diagnosed with PC survive longer the earlier the disease is diagnosed. A summary of survival from $\mathrm{PC}^{2}$ showed that whilst the 5-year survival was about $80 \%$, this varied significantly by stage; with men with metastatic disease at diagnosis having a 5-year survival of around 30\%. Prostate specific antigen (PSA) has been used as a serum based marker for PC detection and monitoring since the 1980 's, but lack of specificity and sensitivity have meant its use as a screening tool in the general population is controversial, and not currently recommended in either the US or the UK ${ }^{3,4}$.

There is therefore still an urgent need for non-invasive biomarkers for the detection of PC in general, and particularly in high-risk populations. Increased risk of PC has been associated with a positive family history, specific single nucleotide polymorphisms (SNPs) identified through genome wide association studies (GWAS) ${ }^{5}$ and deleterious mutations in the DNA repair gene $B R C A 2^{6}$. A number of studies have investigated the relationship between BRCA1 mutations and prostate cancer risk, although there may still remain some controversy regarding this it seems likely that $B R C A 1$ mutations confer increased risk in young onset prostate cancer cases ${ }^{7,8}$. The IMPACT study (Identification of Men with a genetic predisposition to ProstAte Cancer: Targeted Screening in $B R C A 1 / 2$ mutation carriers and controls) was set up to prospectively assess the influence of $B R C A$ status of the development and biology of $\mathrm{PC}$ and thus healthy men were recruited, either carriers of mutated BRCA1 or BRCA2 genes or non-carrier controls, and were screened annually with PSA, using a cut-off of $>3.0 \mathrm{ng} / \mathrm{ml}$ to trigger a diagnostic biopsy'. 
Table 1 | Summary of participant characteristics

\begin{tabular}{lccc} 
& BRCA1/2 mutation carriers & Controls & Pvalue \\
\hline $\mathrm{N}$ & 267 & 140 & 0.106 \\
Mean age (range) & 53.0 years $(40-69)$ & $134 / 140(95.7)$ & 0.665 \\
Caucasion (\%) & $253 / 266(95.1)$ & $56 / 138(40.6)$ & 0.381 \\
Current or ex-smokers (\%) & $95 / 263(36.1)$ & $40 / 136(29.4)$ & 0.469 \\
$>14$ units alcohol/week (\%) & $64 / 257(24.9)$ & $104 / 122(85.0)$ & 0.621 \\
BMl $>25(\%)$ & $199 / 244(81.6)$ & $0 \mathrm{ng} / \mathrm{ml}(0-3045)$ & 0.717 \\
Median EN2 (range) & $0 \mathrm{ng} / \mathrm{ml}(0-3694)$ & $15(10.7)$ & 0.485 \\
Prostate biopsy undertaken (\%) & $35(13.1)$ & $5(3.6)$ & 0.548 \\
Prostate Cancer diagnosed (\%) & $16(6.0)$ & $3(60)$ & 0.375 \\
Tumour T stage $\leq$ T2a (\%) & $6(37.5)$ & & \\
\hline
\end{tabular}

Engrailed-2 (EN2) is a member of the HOX gene family, a subgroup of the homeobox superfamily important in embryonal development. EN2 expression has been described in breast cancer and $\mathrm{PC}^{10}$. We have previously reported the potential diagnostic utility of $\mathrm{EN} 2{ }^{11}$, which we found to be detectable in the urine of PC patients without the requirement for prior DRE. The presence of EN2 in urine was predictive of PC, with a sensitivity of $66 \%$ and a specificity of $88.2 \%$. In a recent retrospective study, a strong positive correlation was shown between pre-surgical levels of urinary EN2 and cancer volume in prostatectomy specimens as well as a correlation between EN2 levels and tumour stage ${ }^{12}$.

The aim of this study was to assess the potential of urinary EN2 for the early diagnosis of PC in the IMPACT study population.

\section{Results}

Participant characteristics. The total number recruited to IMPACT at the time of this study was 1140 from which we selected 418 individuals at random; the most recently donated urine samples were used for EN2 measurement. In five cases it was not possible to obtain a result from the ELISA so these cases were excluded, as were 6 cases who were pending genetic testing, therefore results from 407 participants were reported in the study; the demographic, molecular and pathological characteristics of the participants are shown in table 1 .

The control group comprised 140 men who had received a negative test result for BRCA1 or BRCA2 mutations known to be carried within their family and were therefore considered to be at no higher risk at developing PC than the general population. As shown in table 1 there was no statistical difference in the demographics between the control group and the BRCA1/2 mutation carriers, furthermore EN2 levels did not significantly differ between the two groups. 50 individuals (12.3\%) had undergone prostate biopsy. PC had been diagnosed in 21 men, 9 of these with Gleason $3+3 ; 6$ with Gleason $3+4$ and 6 with Gleason $4+3$. All urine samples pre-dated the diagnosis of cancer.

The investigators were blinded both to cancer status and $B R C A$ mutation carrier status. Table 2 summarises the BRCA1/2 status and Gleason score of the 21 men diagnosed with cancer men; BRCA2 mutation carriers tended towards more aggressive tumours. As per the protocol, all men were biopsied on the basis of a PSA greater than $3 \mathrm{ng} / \mathrm{ml}$. The median PSA amongst the men diagnosed with cancer was $4.3 \mathrm{ng} / \mathrm{ml}$ (range 3.03 to $14.3 \mathrm{ng} / \mathrm{ml}$ ); 17 of the 21 cancers were

\begin{tabular}{|cccc}
\hline \multicolumn{1}{l}{ Table 2 | Gleason score by genetic status } & \\
& Gleason $3+3$ & Gleason $3+4$ & Gleason $4+3$ \\
\hline BRCA1 & 5 & 1 & \\
BRCA2 & 2 & 4 & 4 \\
Controls & 2 & 1 & 2 \\
\hline
\end{tabular}

diagnosed at the initial, prevalence PSA screen so there is no available information on PSA dynamics prior to diagnosis for these men.

EN2 secretion in participants. We measured EN2 in the urine samples from BRCA1/2 mutation carriers and controls from the IMPACT cohort. We found EN2 levels were significantly higher in those diagnosed with cancer compared with those with no diagnosis of cancer, $\mathrm{p} \leq 0.001$. The median EN2 level in those with cancer was $105 \mathrm{ng} / \mathrm{ml}$ (range 0 to $2222 \mathrm{ng} / \mathrm{ml}$ ) versus $0 \mathrm{ng} / \mathrm{ml}$ (range 0 to $3964 \mathrm{ng} / \mathrm{ml}$ ) in the group without cancer. The receiver operating characteristic area under the curve (ROC AUC) for test performance was 0.816 , with a sensitivity of $66.7 \%$ and a specificity of $89.3 \%$ (see table 3 ). The results of the multivariate logistic regression analysis are summarised in table 4, EN2 was a significant independent variable associated with cancer status $(\mathrm{p}<0.001)$. The positive predictive value (PPV) of EN2 was 25\%, which increased to $73.7 \%$ when only those biopsied were analysed. Of the 42 men with EN2 level $>42.5 \mathrm{ng} / \mathrm{ml}$ in their urine who had not been diagnosed with cancer, only 5 had undergone prostate biopsy so the true cancer status of the remaining 37 individuals is unknown. Of all the 364 men who had not undergone biopsy (as their PSA had not exceeded $3.0 \mathrm{ng} / \mathrm{ml}$ ) $10.2 \%$ secreted $>42.5 \mathrm{ng} / \mathrm{ml} \mathrm{EN} 2$ in their urine.

PSA findings. The sensitivity of PSA to detect cancer could not be calculated in this cohort as patients underwent prostate biopsy based on elevated PSA (although there have been no symptomatic interval cancers), therefore by definition sensitivity was $100 \%$. The specificity of PSA in this study was $91.3 \%$. The PPV of PSA was $38.9 \%$ which increased to $53.8 \%$ when only those biopsied were analysed. A very weak correlation was found between EN2 and PSA, Spearman's rank correlation coefficient was 0.138 (see figure 1 ).

Effect of BRCA1/2 mutation status. There was no significant difference in EN2 levels between BRCA1/2 mutation carriers, and controls $(\mathrm{p}=0.717)$. EN2 performed with the highest sensitivity in those with BRCA2 mutations (see table 5), however the numbers involved are very small and these differences may be observed by chance. As shown in table 2, those with BRCA2 mutations tended toward more aggressive cancers which may be an explanation for the better performance of EN2 as a biomarker in this group. Logistic regression analysis showed $B R C A 1 / 2$ mutation status was not a significant variable in predicting cancer outcome (see table 4).

Table 3 | Summary of EN2 results by cancer status

\begin{tabular}{lccc|} 
& Cancer & No Cancer & \\
\hline EN2 positive & $14(66.7 \%)$ & $42(10.7 \%)$ & 56 \\
EN2 negative & $7(33.3 \%)$ & $350(89.3 \%)$ & 397 \\
& 21 & 392 & 413
\end{tabular}


Table 4 | Logistic regression results for the effect of EN2 and $B R C A 1 / 2$ status on cancer status

\begin{tabular}{llrc} 
Variable & Estimate $(\beta)^{\dagger}(95 \% \mathrm{Cl})$ & P value & Odds ratio $\left(\mathrm{e}^{\beta}\right)$ \\
\hline EN2* & $2.7877(1.82-3.75)$ & $<0.001$ & 16.24 \\
Genetic status & $0.1349(-0.92-1.19)$ & 0.798 & 1.14 \\
\hline
\end{tabular}

Coefficient associated with each variable.

EN2 analysed as a binary variable, using $42.5 \mathrm{ng} / \mathrm{ml}$ as cut-off.

Gleason score and stage. When the cancer cases were divided into those with Gleason $\leq 3+4$ versus those with Gleason $\geq 4+3$ there was no significant difference in EN2 levels $(p=0.651)$. Using the NICE guidance for risk stratification ${ }^{13}$ there were seven low risk cases, 12 intermediate risk and two high risk cases. There was no significant difference in EN2 level between these three groups ( $\mathrm{p}=$ $0.634)$.

\section{Discussion}

Men with mutations in $B R C A 1$ and $B R C A 2$ genes are at increased risk of $P C$ with a 3.5 -fold and 8.6 -fold increase respectively ${ }^{6,7}$; furthermore cancer is more likely to develop at a younger age, and to be more aggressive $e^{9,14}$. This makes BRCA mutation carriers an ideal group for 'targeted' screening as early detection and treatment of PC would be expected to improve their prognosis.

Although PSA has high clinical utility for the management of PC, its reliability as a screening tool is less certain. For the general public, PSA screening is not justified on the basis of limited sensitivity and specificity $^{15}$. For the IMPACT protocol, a PSA level of $>3.0 \mathrm{ng} / \mathrm{ml}$ was selected as the threshold to proceed to biopsy. The purpose of this study was to determine whether urinary EN2 secretion serves as a useful biomarker for the early detection of PC.

The EN2 urinary biomarker has previously been demonstrated not only to be able to detect PC in $70-85 \%$ of men with the disease, but also to correlate strongly with tumour volume in a radical prostatectomy population ${ }^{16}$. Tumour volume is important in stratification of clinical significance of PC; it has been shown that small volume disease $(<0.5 \mathrm{mls})$ may be 'insignificant' and may not require treatment but would be more suitable for active surveillance $^{17}$. In the group of men in our IMPACT study with histologically proven PC, the sensitivity and specificity of detection was similar to the previously published studies ${ }^{11,16}$ with $66.7 \%$ of those with cancer having EN2 detected in their urine. Of the seven cancer cases deemed negative for urinary EN2, it is not known whether EN2 is expressed but not secreted into the urine, or simply not expressed by the tumour tissue.

We found significantly elevated levels of EN2 in 42 men who otherwise did not have any clinical signs of PC. Five of these men had a PSA rise which led to prostatic biopsy by the study protocol, and these were all negative for cancer. All biopsies performed in the study were transrectal ultrasound (TRUS) guided ten core sampling (with a further two cores taken for research purposes). This is an accepted biopsy approach for men with suspected PC, cores are targeted to the peripheral zone from which the majority of cancers arise, however a significant number of cancers may be still be missed by this method ${ }^{18}$. Newer techniques such as 36 core transperineal template biopsy are being introduced for the detection of cancer in men in whom there is a high clinical suspicion of PC but a negative TRUS biopsy ${ }^{19}$. Therefore it is possible that the five men described may still be harbouring PC despite these negative biopsies.

It is not known how many cancers may remain undetected in the $37(8.9 \%)$ men with a positive urinary EN2 but who had not undergone biopsy as PSA $\leq 3.0 \mathrm{ng} / \mathrm{ml}$. Data from Thompson et $\mathrm{al}^{20}$ showed that 397 of 2757 men (14.4\%) with PSA $\leq 3.0 \mathrm{ng} / \mathrm{ml}$ were diagnosed with PC, and $13.6 \%$ of these were high grade (defined as Gleason $\geq 7$ ), however the age range in that study was 62 to 91 years, considerably older than the IMPACT cohort. A smaller study evaluating men with a family history of PC, age range 39 to 80 years found 15 of 78 men (19.2\%) with PSA $\leq 3.0$ were diagnosed with $\mathrm{PC}^{21}$. We may expect a higher rate still, in this genetically high risk group. The ability of EN2 to detect PC independent of PSA level is supported by one individual who was diagnosed with PC 3 years into the study when his PSA measured $3.3 \mathrm{ng} / \mathrm{ml}$, however the EN2 level in his urine sample from his initial study visit was elevated at $144 \mathrm{ng} / \mathrm{ml}$ when his PSA measured $2.2 \mathrm{ng} / \mathrm{ml}$. It is possible the remaining 37

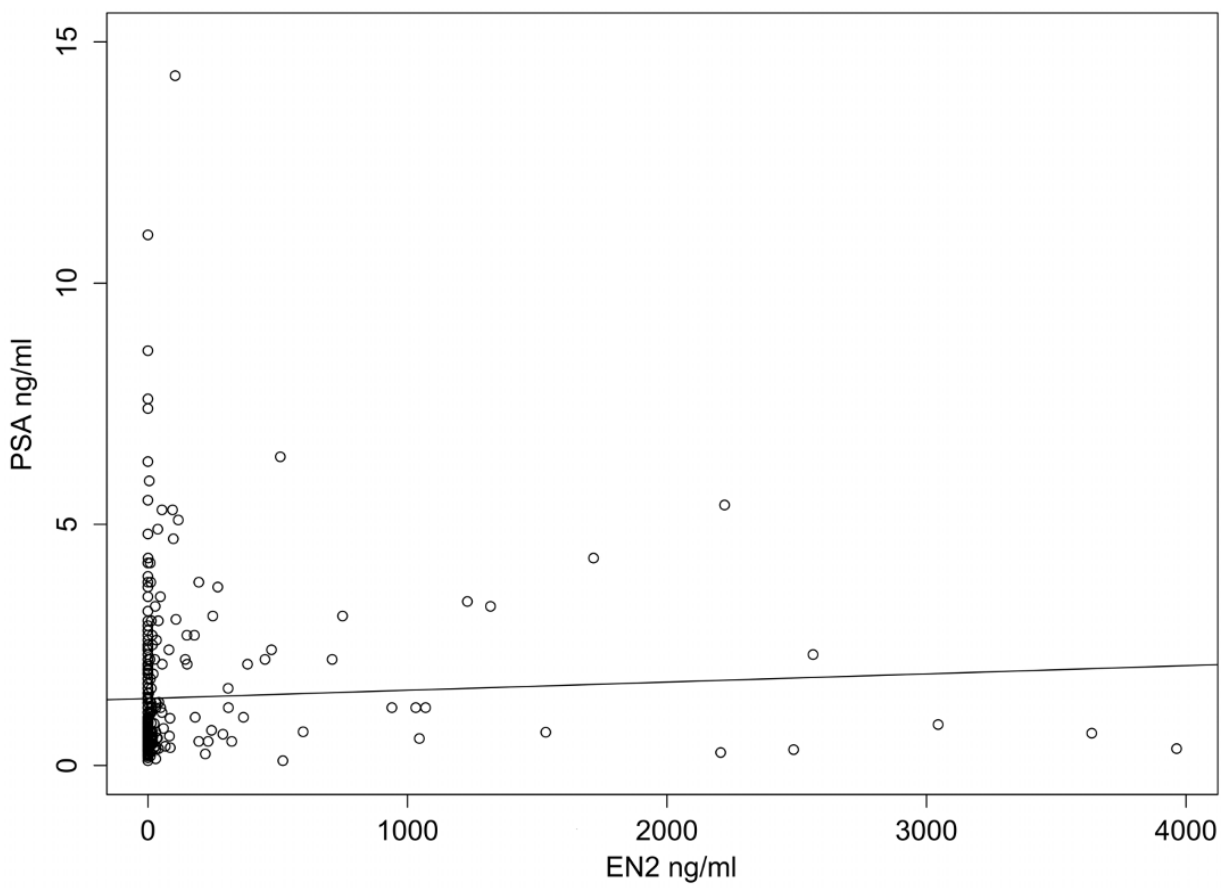

Figure $1 \mid$ Association between serum PSA and urinary EN2. 
Table 5 | Effect of BRCA mutation status on sensitivity and specificity of EN2

\begin{tabular}{lllllll|} 
& \multicolumn{2}{c}{ BRCA1 } & \multicolumn{2}{c}{ BRCA2 } & \multicolumn{2}{c}{ Controls } \\
\hline & cancer & no cancer & cancer & no cancer & cancer & no cancer \\
\cline { 2 - 7 } EN2 positive & $4(66.7 \%)$ & 13 & $8(80 \%)$ & 16 & $2(40 \%)$ & 13 \\
EN2 negative & 2 & $110(89.4)$ & 2 & $112(87.5 \%)$ & 3 & $122(90.4 \%)$ \\
\hline
\end{tabular}

men with high EN2 levels but PSA $<3.0 \mathrm{ng} / \mathrm{ml}$ are harbouring occult PC.

Direct comparisons between the utility of PSA and EN2 in this cohort are biased by the use of PSA to determine who underwent prostate biopsy. The PSA sensitivity was $100 \%$ as would be expected where no patients with a PSA $<3.0 \mathrm{ng} / \mathrm{ml}$ underwent biopsy. The specificity of PSA was $91.6 \%, 33$ of the 392 individuals who were not diagnosed with PC had a PSA $>3.0 \mathrm{ng} / \mathrm{ml}$. It should be noted that although all these men were offered prostate biopsy 15 of them declined. In the general population a PSA cut-off of $3.0 \mathrm{ng} / \mathrm{ml}$ has been shown to have a sensitivity of $32.2 \%$ and a specificity of $86.7 \%{ }^{22}$, although there may be evidence that PSA performs better as marker for cancer screening in BRCA1/2 mutation carriers?. The PPV for both EN2 and PSA was low $38.9 \%$ and $25.0 \%$ respectively. PPV improved when analysis was restricted to those men who underwent prostate biopsy, particularly for EN2 which increased to $73.7 \%$ (53.8\% for PSA). Although EN2 has previously been reported to have no correlation with $\mathrm{PSA}^{12}$ we found a weak correlation with Spearman's rank correlation coefficient of 0.138 .

Given the small numbers involved in the study, sub-group analysis by $B R C A$ mutation carrier status is not possible, however there is a suggestion that EN2 performs best in men with BRCA2 mutations, and it is this group which have the highest risk ${ }^{6}$.

A limitation of this study is the significant proportion (87.9\%) of patients who have not undergone prostate biopsy. The IMPACT study protocol includes annual PSA screening for 5 years at which point recruits are offered an optional prostate biopsy. Currently the first few recruits are reaching the 5 year time point and approximately $50 \%$ are opting to proceed with the biopsy. We predict that over the next 5 years approximately half of the 368 individuals with PSA $<3.0 \mathrm{ng} / \mathrm{ml}$ will undergo prostate biopsy, and this study should therefore be considered as an interim study providing us with preliminary information about the operating characteristics of EN2.

EN2 represents a potentially attractive biomarker for early detection of cancer. As a urine-based test stable for up to 4 days at room temperature it is suitable for the postal collection of samples. Unlike some other urinary markers such as PCA3 and TMPRSS2-ERG, prior DRE is not required improving the acceptability to individuals and reducing cost. Lateral flow strips are currently in commercial development which will improve the speed and ease of investigation of EN2 as an early, point of care cancer detection marker. The IMPACT study is expected to complete recruitment of BRCA1 and $B R C A 2$ carriers and controls by the end of 2013, a further five year follow-up is expected to give further information on the utility of PSA screening in this high risk cohort as well as providing a useful sample bank for the investigation of EN2 and other novel detection or prognostic biomarkers for PC. As more recruits reach the 5 year point when optional prostate biopsy is offered regardless of PSA further information will be available regarding true cancer status of these individuals and effectiveness of EN2 as an early biomarker for PC.

\section{Methods}

Patients. Urine samples were taken from the IMPACT study, an international study designed to evaluate PSA screening in male BRCA1 and BRCA2 carriers. The experiments were approved by a Research Ethics Committee (MREC reference number 05/MRE07/25); all participants gave informed written consent. Recruitment to this study began in 2005, and is on-going. Eligible men were aged 40-69 with no history of PC, and had had no previous biopsy for raised PSA. All individuals were from families known to carry pathogenic $B R C A 1$ or $B R C A 2$ mutations although it was not necessary for individuals to know their genetic status prior to enrolment, they could be tested while on study. The case group consisted of men who carried pathogenic $B R C A 1$ and $B R C A 2$ mutations. The control group comprised men who had received a negative test result for $B R C A 1$ or $B R C A 2$ mutations found within their family. Exclusion criteria included previous history of PC or prostate biopsy. Recruits underwent baseline then annual serum PSA screening with PSA $>3.0 \mathrm{ng} / \mathrm{ml}$ triggering a diagnostic 10 core transrectal ultrasound (TRUS) guided biopsy of the prostate. Where prostate cancer was detected in the biopsy sample, this was graded according to Gleason Score, a system whereby two numbers are assigned, $a+b$ where a represents the most common pathological grade of tumour in the sample and $b$ represents the second most common grade (of which there must be more than 5\%). Each score ranges from one to five with five being the most aggressive, however in practice scores below three are not used these two score can then be added together to give a Gleason sum $(\mathrm{eg} \mathrm{Gl} 3+4=7)^{23,24}$. Urine and serum samples were collected annually, aliquotted into $1.8 \mathrm{ml}$ Nunc Cryotubes and transferred to $-80^{\circ} \mathrm{C}$ freezer within 4 hours.

EN2 ELISA test Urinary EN2 levels were tested by ELISA following a previously published method ${ }^{11}$. Briefly, two monoclonal anti-EN2 antibodies were raised, APS1 and APS2 (Antibody Production Services Ltd, Haywards Heath, UK); the antigen used was a synthetic protein corresponding to the C-terminal 100 amino acids of EN2 (Biosynthesis Inc, Texas, USA). APS2 was conjugated to biotin and captured onto a 96 well streptavadin coated plate (Nunc 436014, New York, USA).

EN2 dilutions were prepared in reference urine from an individual unaffected by PC whose urine was negative for EN2, with 0.1 vols $10 \times$ phosphate buffered saline tween (PBST) added (giving 90\% reference urine in PBST). A set of serial dilutions were prepared, and $100 \mu \mathrm{l}$ of these EN2 standards were added to appropriate wells in duplicate. $90 \mu \mathrm{l}$ of test urine mixed with $10 \mu \mathrm{l}$ of $10 \times$ PBST was added to appropriate wells in duplicate.

The APS1 antibody was conjugated to alkaline phosphatase. APS1-Alkaline phosphatase detection antibody was prepared at 1:250 dilution in PBST, and added to appropriate wells. Colometric agent p-nitrophenyl phosphate substrate was added, incubated and absorbance read at $405 \mathrm{~nm}$ using a Beckman-Coulter DX880. The dilution series was used to generate a standard curve from which the concentration of EN2 could be read. Any runs where the standard curve had an $\mathrm{R}^{2}<0.95$ were repeated. An EN2 level of $>42.5 \mathrm{ng} / \mathrm{ml}$ was deemed as positive, this cut-off was taken from previously published work $^{11}$.

Statistical analysis sensitivity and specificity were calculated for detection of cancer by EN2, and ROC AUC calculated. Logistic regression was used to examine associations between EN2, genetic status and outcome (cancer diagnosis). To test the significance of differences in EN2 levels in different patient groups (by cancer status and Gleason status) we used the independent samples Mann-Whitney U test. In order to test significance of differences in EN2 according to genetic status we used the Kruskall-Wallis one-way analysis of variance by ranks. Spearman's rank correlation coefficient was used to measure correlation between PSA and EN2 levels.

1. Siegel, R., Naishadham, D. \& Jemal, A. Cancer statistics, 2012. CA Cancer JClin 62, 10-29 (2012).

2. South West Public Health Observatory. SWPHO Briefing: Prostate cancer survival by stage, $<\mathrm{http}: / /$ www.swpho.nhs.uk/resource/item.aspx?RID $=41287>(2008)$. Date accessed 15.07.11.

3. Andriole, G. L. et al. Mortality results from a randomized prostate-cancer screening trial. N Engl J Med 360, 1310-1319 (2009).

4. Schroder, F. H. et al. Screening and prostate-cancer mortality in a randomized European study. N Engl J Med 360, 1320-1328 (2009).

5. Goh, C. L. et al. Genetic variants associated with predisposition to prostate cancer and potential clinical implications. J Intern Med 271, 353-365 (2012).

6. Kote-Jarai, Z. et al. BRCA2 is a moderate penetrance gene contributing to youngonset prostate cancer: implications for genetic testing in prostate cancer patients Br J Cancer 105, 1230-1234 (2011).

7. Leongamornlert, D. et al. Germline BRCA1 mutations increase prostate cancer risk. Br J Cancer 106, 1697-1701 (2012).

8. Fachal, L. et al. BRCA1 mutations do not increase prostate cancer risk: results from a meta-analysis including new data. Prostate 71, 1768-1779 (2011).

9. Mitra, A. V. et al. Targeted prostate cancer screening in men with mutations in BRCA1 and BRCA2 detects aggressive prostate cancer: preliminary analysis of the results of the IMPACT study. BJU Int 107, 28-39 (2011).

10. Martin, N. L., Saba-El-Leil, M. K., Sadekova, S., Meloche, S. \& Sauvageau, G. EN2 is a candidate oncogene in human breast cancer. Oncogene 24, 6890-6901 (2005). 
11. Morgan, R. et al. Engrailed-2 (EN2): a tumor specific urinary biomarker for the early diagnosis of prostate cancer. Clin Cancer Res 17, 1090-1098 (2011).

12. Pandha, H. et al. Urinary engrailed-2 (EN2) levels predict tumour volume in men undergoing radical prostatectomy for prostate cancer. BJU Int 110, E287-292 (2012).

13. NICE. Prostate cancer: Diagnosis and treatment, <www.nice.org.uk/CG58> (2008). Date accessed 28.06.12.

14. Mitra, A. et al. Prostate cancer in male BRCA1 and BRCA2 mutation carriers has a more aggressive phenotype. Br J Cancer 98, 502-507 (2008).

15. USPSTF. Screening for prostate cancer: draft recommendation statement. Rockville, MD: U.S. Preventive Services Task Force, < http:// www.uspreventiveservicestaskforce.org/prostatecancerscreening/ draftrecprostate.htm $>$ (2011). Date accessed 13.03.13.

16. Pandha, H. et al. Urinary engrailed-2 (EN2) levels predict tumour volume in men undergoing radical prostatectomy for prostate cancer. BJU Int (2012).

17. Epstein, J. I. et al. Nonpalpable stage T1c prostate cancer: prediction of insignificant disease using free/total prostate specific antigen levels and needle biopsy findings. J Urol 160, 2407-2411 (1998).

18. Norberg, M. et al. The sextant protocol for ultrasound-guided core biopsies of the prostate underestimates the presence of cancer. Urology 50, 562-566 (1997).

19. Pal, R. P., Elmussareh, M., Chanawani, M. \& Khan, M. A. The role of a standardized 36 core template-assisted transperineal prostate biopsy technique in patients with previously negative transrectal ultrasonography-guided prostate biopsies. BJU Int 109, 367-371 (2012).

20. Thompson, I. M. et al. Prevalence of prostate cancer among men with a prostatespecific antigen level $<$ or $=4.0 \mathrm{ng}$ per milliliter. $N$ Engl J Med 350, 2239-2246 (2004).

21. Canby-Hagino, E. et al. Prostate cancer risk with positive family history, normal prostate examination findings, and PSA less than $4.0 \mathrm{ng} / \mathrm{mL}$. Urology 70, 748-752 (2007).

22. Thompson, I. M. et al. Operating characteristics of prostate-specific antigen in men with an initial PSA level of $3.0 \mathrm{ng} / \mathrm{ml}$ or lower. JAMA 294, 66-70 (2005).

23. Shah, R. B. Current perspectives on the Gleason grading of prostate cancer. Arch Pathol Lab Med 133, 1810-1816 (2009).

24. Gleason, D. F. \& Mellinger, G. T. Prediction of prognosis for prostatic adenocarcinoma by combined histological grading and clinical staging. J Urol 111, 58-64 (1974).

\section{Acknowledgements}

We would like to thank the IMPACT study participants and their families. We would also like to thank the IMPACT collaborators from around the world. We are grateful to Tokhir Dadaev for statistical advice. We acknowledge support from the Annabel Evans Memorial Fund, the Ronald \& Rita McAulay Foundation, the Prostate Project Charity, Basser Research Centre, Cancer Research UK (grant numbers C5047/A8385 and C5047/A13232) and NIHR. We also acknowledge financial support in Australia for IMPACT from the Cancer Councils of Victoria and South Australia, grant number 400048, the Prostate Cance Foundation of Australia grant number PCFA PRO4 and Cancer Australia grant number 1006349. We acknowledge funding from Jack and Judy Baker for the study at NorthShore University HealthSystem, Evanston, Illinois, and Myriad Genetics Laboratory, Salt Lake City, Utah, for providing research BRCA testing rates for NorthShore University HealthSystem participants.

\section{Author contributions}

The manuscript was prepared by E.K. with input from E.K.B., Z.K.J., H.P. and R.E.; al authors reviewed the manuscript. The EN2 ELISA assay was developed by H.P. and R.M. and run by R.M., F.L. and E.K. Sample collection and processing was done by E.K.B., E.P., E.C. and E.K., A.A., I.B., V.C., S.D., F.D., D.E., G.E., M.H., J.K., J.L., G.L., G.M., N.P., C.S., K.T., J.Z. and R.E. recruited the patients to IMPACT who provided the urine samples. H.P. and R.E. had oversight of the scientific strategy with support from R.M., Z.K.J. and E.K.

\section{Additional information}

Competing financial interests: The authors declare no competing financial interests.

How to cite this article: Killick, E. et al. Role of Engrailed-2 (EN2) as a prostate cancer detection biomarker in genetically high risk men. Sci. Rep. 3, 2059; DOI:10.1038/srep02059 (2013)

(i) $\mathrm{T}$ This work is licensed under a Creative Commons Attribution-

By nc No NonCommercial-NoDerivs 3.0 Unported license. To view a copy of this license, visit http://creativecommons.org/licenses/by-nc-nd/3.0 